\title{
Hospital-level associations with 30-day patient mortality after cardiac surgery: a tutorial on the application and interpretation of marginal and multilevel logistic regression
}

\author{
Masoumeh Sanagou*, Rory Wolfe, Andrew Forbes and Christopher Michael Reid
}

\begin{abstract}
Background: Marginal and multilevel logistic regression methods can estimate associations between hospital-level factors and patient-level 30-day mortality outcomes after cardiac surgery. However, it is not widely understood how the interpretation of hospital-level effects differs between these methods.

Methods: The Australasian Society of Cardiac and Thoracic Surgeons (ASCTS) registry provided data on 32,354 patients undergoing cardiac surgery in 18 hospitals from 2001 to 2009. The logistic regression methods related 30day mortality after surgery to hospital characteristics with concurrent adjustment for patient characteristics.

Results: Hospital-level mortality rates varied from $1.0 \%$ to $4.1 \%$ of patients. Ordinary, marginal and multilevel regression methods differed with regard to point estimates and conclusions on statistical significance for hospitallevel risk factors; ordinary logistic regression giving inappropriately narrow confidence intervals. The median odds ratio, MOR, from the multilevel model was 1.2 whereas ORs for most patient-level characteristics were of greater magnitude suggesting that unexplained between-hospital variation was not as relevant as patient-level characteristics for understanding mortality rates. For hospital-level characteristics in the multilevel model, $80 \%$ interval ORs, IOR-80\%, supplemented the usual ORs from the logistic regression. The IOR- $80 \%$ was (0.8 to 1.8) for academic affiliation and (0.6 to 1.3) for the median annual number of cardiac surgery procedures. The width of these intervals reflected the unexplained variation between hospitals in mortality rates; the inclusion of one in each interval suggested an inability to add meaningfully to explaining variation in mortality rates.
\end{abstract}

Conclusions: Marginal and multilevel models take different approaches to account for correlation between patients within hospitals and they lead to different interpretations for hospital-level odds ratios.

\section{Background}

Over the past two decades there has been a dramatic growth in the publication of cardiac surgery outcomes research. Many recent studies have examined the impact of hospital, physician, and process-related characteristics on outcomes for hospitalized patients who have undergone cardiac surgery [1-8]. By virtue of their observational design, these studies rely heavily on the use of regression modelling to remove the effects of confounding variables [9-11]. Data from these studies usually have

\footnotetext{
* Correspondence: Masoumeh.Sanagou@monash.edu

Department of Epidemiology and Preventive Medicine, Faculty of Medicine, Nursing and Health Sciences, Monash University, Melbourne, Australia
}

a two-level structure of patients within hospitals, a familiar structure in epidemiological studies [12,13].

It is generally recognised in many areas of the social, medical and other sciences that data arise in complex multilevel structures, for example responses from individuals who are grouped together in communities or institutions. An understanding of appropriate analytical methods is vital for researchers in fields such as education, epidemiology, geography, child growth and social surveys, among others. There is a rich literature on analytical methods for two-level data structures with particular emphasis on multilevel [14-17] and marginal [14,17] models. These methods have subtle differences in interpretation when applied
Ciomed Central 
in different multilevel contexts, for example to longitudinal studies [18,19], to cluster randomised trials [20], or, as focussed on here, to observational studies in which individual responses are correlated due to a shared environment or process the nature of which may only in part be measurable.

Ordinary (single level) logistic regression is usually inappropriate for patient-within-hospital outcomes because it assumes all outcomes are independent $[21,22]$. Patients within a given hospital typically tend to be more alike than patients across different hospitals in measured and unmeasured characteristics predictive of outcome, for example socio-economic status. Further, at the hospital-level, the implementation of specific quality assurance programs (such as treatment protocols and critical care maps) may result in less heterogeneity in the use of evidence-based therapies for patients admitted to a particular hospital [23]. Ignoring the clustering present in multilevel data, as occurs in ordinary logistic regression, results in an artificially inflated number of independent observations at the hospital level of the hierarchy, and hence is likely to underestimate the magnitude of the standard error for the effect of hospital-level characteristics. Marginal and multilevel statistical techniques have been developed to deal with data arranged in a natural hierarchy $[15,16,24]$. Ordinary and multilevel models have been used to investigate whether the choice of statistical methods affects which hospitals are classified as high- and low-performance outliers in the publicly available data at the New York State, Department of Health, Coronary Artery Bypass Graft (CABG) Surgery Report Card [25]. In the context of patients-in-hospitals two-level data, marginal [26] and multilevel logistic regression $[27,28]$ have been used to examine the impact of risk factors on outcomes when adjusting for differences in patient and hospital characteristics. The interpretation of effect estimates differs for marginal and multilevel models for binary outcomes and this is understood when interpreting patient-level risk factors $[29,30]$. Not so widely appreciated is how the interpretation of hospital-level effects also differs between these models. Further, multilevel logistic regression offers additional parameters, the median odds ratio (MOR) [31,32], and $80 \%$ interval odds ratios (IOR-80\%) [31,32], that can help to shed light on hospital-to-hospital variability in outcome and the impact of hospital-level risk factors, respectively.

The aim of this study was to apply ordinary, marginal and multilevel logistic regression models to 30-day mortality outcomes of 32,354 patients in the Australasian Society of Cardiac and Thoracic Surgeons (ASCTS) registry in order to compare the three methods with the focus on the interpretation of hospital-level risk factors.

\section{Methods}

\section{Patient population and data}

The Australasian Society of Cardiac and Thoracic Surgeons (ASCTS) is responsible for a registry of cardiac and thoracic surgery in Australia covering nearly half of the country's private and public cardiothoracic surgical units.

Data was collected on all patients undergoing cardiac surgery between July 2001 and June 2009 in 18 hospitals in Australia. In each hospital, a data manager was responsible for the completeness of the data collection. All data was verified on receipt by the co-ordinating centre which followed up on queries about missing data, outliers or inconsistent reports. The data was validated locally and also by an external data quality audit program. This program was performed on-site to evaluate the completeness (2.4\% missing value) and accuracy of the data collected within the combined database [33].

The ASCTS registry collected information on patient preoperative risk factors (including preoperative cardiac status and previous interventions), intra-operative details (including the procedure performed, myocardial protection and procedural duration), complications and post operative outcomes. In this study, the outcome variable was mortality within 30-days of cardiac surgery. This information was collected by the data managers by contacting medical practitioners, patients or family members by telephone as part of clinical care.

This research project was undertaken following approval from the ASCTS Research Committee which governs access to data from the registry. Ethical approval for the use of de-identified registry data for secondary research purposes such as this project had previously been provided by each participating institution's ethics review committee.

\section{Patient and hospital level characteristics}

Patient-level characteristics in the registry included: age, gender, the New York heart association (NYHA) class, urgency of procedure, ejection fraction estimate, lipidlowering treatment (hypercholesterolaemia), preoperative dialysis, previous cardiac surgery, procedure type, inotropic medication (inotropes), peripheral vascular disease, and body mass index (BMI) [34]. Two hospital-level characteristics were assessed, namely academic affiliation (teaching or non-teaching status) and the median across 2001-2009 of the annual number of cardiac surgeries. Academic affiliation of the 15 teaching hospitals and 3 non-teaching hospitals did not change throughout the study period. All patient and hospital characteristics were included in analysis as categorical risk factors except the median annual number of cardiac surgeries which was continuous. 


\section{Statistical techniques}

\section{Ordinary, marginal and multilevel logistic regression}

Logistic regression [35] was used to assess the influence of risk factors on 30-day mortality. Let $\mathrm{p}_{\mathrm{ij}}$ be the probability and $\frac{p_{i j}}{1-p_{i j}}$ the odds of death for patient $j$ in hospital i. The equation of the ordinary logistic model was

$$
\log \left(\frac{p_{i j}}{1-p_{i j}}\right)=\beta_{0}+\sum_{k=1}^{R} \quad \beta_{k} X_{i j k}
$$

where the $X_{i j k}$ 's represent a patient's values of $R$ risk factors, and $\beta_{1} \ldots \beta_{\mathrm{R}}$ are regression coefficients corresponding to each risk factor. For a given risk factor, its coefficient $\beta_{\mathrm{k}}$ is the log odds ratio corresponding to a 1-unit difference in continuous $\mathrm{X}_{\mathrm{k}}$ or, if a risk factor is an indicator, for example of peripheral vascular disease ( 1 if yes, 0 if no), then $\beta_{\mathrm{k}}$ is the log odds ratio comparing the effect on mortality of the risk factor's presence with its absence. Exponentiating $\beta_{\mathrm{k}}\left(e^{\beta_{k}}\right)$ gives the corresponding odds ratio, OR. In ordinary logistic regression hospital characteristics are treated the same as patient-level risk factors, and patient outcomes $\mathrm{Y}_{\mathrm{ij}}$ are assumed to be independent binomial variables with mortality probability $\mathrm{p}_{\mathrm{ij}}$.

A model based on generalized estimating equations, GEE [36], may also be used for analysis of patient mortality outcomes. For this, equation (1) is combined with the following assumptions: firstly as before the probability of $Y_{i j}=1$, a death, is $\mathrm{p}_{\mathrm{ij}}$ and $\mathrm{Y}_{\mathrm{ij}}$ has binomial variance $\mathrm{p}_{\mathrm{ij}}\left(1-\mathrm{p}_{\mathrm{ij}}\right)$. Secondly, it is assumed that patients within a hospital have correlated outcomes but patient outcomes in different hospitals are independent, i.e. have zero correlation. An exchangeable working correlation structure in the GEE estimation process assumes that pair-wise correlations between patient outcomes within the same hospital are equal and can be represented by the parameter $\rho$.

The GEE method includes the calculation of robust estimates for the standard errors of the regression coefficients that ensure consistent inference even if the chosen working correlation structure is incorrect or if the strength of the correlation between patient outcomes within the same hospital varies somewhat from patient to patient.

Multilevel logistic regression [15] assumes that each hospital has its own underlying mortality probability and this varies from hospital to hospital. Specifically, a logistic regression for patients includes an additional term $u_{i}$, a hospital-level random effect, as a predictor variable:

$$
\log \left(\frac{p_{i j}^{*}}{1-p_{i j}^{*}}\right)=u_{i}+\beta_{0}+\sum_{k=1}^{R} \quad \beta_{k} X_{i j k}
$$

Note that $p_{i j}^{*}$ in this model is the conditional probability that patient $\mathrm{j}$ in hospital $\mathrm{i}$ died, and here the probability depends on the value of the random effect, $u_{i}$, for that hospital. $u_{i}$ is the totality of measured and unmeasured hospital-level variables that predict mortality and are uncorrelated with the individual and hospital-level predictor variables in the model. In other words $u_{i}$ represents the combination of omitted hospital-level variables.

Variation in the mortality propensity between hospitals is accommodated by assuming a normal distribution for $u_{i}$ with mean zero and variance $\tau^{2}$. A hospital with $\mathrm{u}_{\mathrm{i}}=0$ can be thought of as having "average" (compared to other hospitals in the population) mortality probability (on the $\log$ odds scale). Higher values of $\tau^{2}$ indicate greater heterogeneity in mortality among hospitals. By including $u_{i}$ in the model as a random effect, the interdependencies among patients within hospitals are explicitly taken into account.

\section{Odds ratio interpretation in ordinary, marginal and multilevel logistic regression}

The interpretation of the odds ratio for patient-level risk factors $\left(e^{\beta}\right)$ in ordinary logistic and marginal models is the same, but differs from the interpretation in the multilevel logistic model [29]. The marginal models estimate population-averaged (or population-marginalized) parameters [30]. In a marginal model, odds ratios characterize the effect of predictors on the population as a whole, averaged over $u_{i}$, rather than on a typical hospital [30]. Odds ratios in a marginal model represent, across all hospitals, differences in mortality between all patients with one value of a risk factor to all patients with the other value.

In multilevel models, for patient-level variables, the usual odds ratio interpretations apply for comparisons of patients within the same hospital; for example, a body mass index (BMI) effect may be interpreted as an odds ratio between a patient with $\mathrm{BMI}<25$ and a patient with $\mathrm{BMI}>25$ belonging to the same hospital and with the same covariates, except for BMI.

Odds ratios for hospital risk factors in marginal models are interpreted as the odds of mortality for hospitals with one value of the factor compared to hospitals with another value of the factor. For example, the odds of 30-day mortality for non-teaching hospitals compared to teaching hospitals.

However, the odds ratio for a hospital-level risk factor in multilevel logistic regression has a different interpretation, namely the odds of mortality for hospitals with one value of the factor compared to hospitals with another value of the factor but with the same value of random effect. For example, the odds of 30-day mortality for a patient treated at a non-teaching hospital 
compared to a patient treated at a teaching hospital with the same value of $u_{i}$. Because of this difficult interpretation an additional parameter, the $80 \%$ interval odds ratio (IOR-80\%), has been developed and is described later in this paper.

The OR for a risk factor in a marginal model is adjusted for the other risk factors included in $\mathrm{X}_{\mathrm{ijk}}$. In a multilevel model the ORs are additionally adjusted for unobserved hospital-level characteristics via the random effect. Due to a mathematical property called non-collapsibility of the odds ratio, the odds ratio for a risk factor from a multilevel model is likely to be further from the null value of one than the odds ratio for that risk factor from a marginal model [37].

\section{Intra-class correlation coefficient (ICC)}

The fundamental reason for applying special statistical techniques in multilevel analysis is the likely existence of intra-class (intra-hospital) correlation arising from similarity of mortality risk of patients of the same hospital compared to those of different hospitals. Patients operated on at the same hospital may be more similar to each other than patients operated on in other hospitals, as they share a number of economic, social, and other characteristics that may condition similar health status beyond what can be adjusted for by patient-level covariates.

The total variance in the outcome variable is the sum of patient-level and hospital-level variances. In multilevel logistic regression however, the patient-level variance is on the probability scale whereas the hospital-level variance is on the logistic scale. To solve this technical difficulty the linear threshold approximation has been proposed [16] and its solution is to convert the patientlevel variance from the probability scale to the logistic scale. The method assumes that the propensity to die is a continuous latent variable and only those patients whose propensity crosses a certain threshold will die as defined by the binary outcome. The unobserved patient variable follows a logistic distribution with patient-level variance equal to 3.29 . On this basis, the ICC is calculated as:

$$
I C C=\frac{\tau^{2}}{\tau^{2}+3.29}
$$

where $\tau^{2}$ is the estimated variance of the random effect of hospital.

In the marginal model, the working correlation structure is the mechanism that accounts for ICC, and the parameter $\rho$ can be interpreted as a measure of intrahospital correlation in mortality outcomes. There is a subtle distinction between $\rho$ and the ICC defined in (3) for the conceptual propensity-to-die variable. It has been shown that $\rho$ in a marginal model is smaller than
ICC in the corresponding multilevel model when there are a small number of clusters [38].

For a binary outcome like mortality, the term ICC can be difficult. Conceptual problems with the ICC (it is a concept from linear regression that has no exact equivalent for logistic regression), interpretational issues as outlined above, and generalisability problems (ICC depends on outcome prevalence) are some of its limitations. Similarly, $\tau^{2}$, the inter-hospital variation in mortality, is difficult to interpret because it is on a log-odds scale $[39,40]$.

\section{The median odds ratio (MOR)}

The MOR is potentially easier to interpret than the ICC because it expresses inter-hospital variance on the OR scale, on which the effects of risk factors are also interpreted.

MOR is defined for a multilevel model as the median of the set of odds ratios that could be obtained by comparing two patients with identical patient-level characteristics from two randomly chosen, different hospitals, i.e. different in hospital random effect value [31,32]. The MOR is the median odds ratio between the patient in the hospital with higher mortality propensity and the patient in the hospital with lower mortality propensity.

The MOR is a measure of variation between the mortality rates of different hospitals that is not explained by the modelled risk factors. The MOR can be shown [41] to be simply related to $\tau^{2}$ as

$$
M O R=\exp \left[\sqrt{2 \times \tau^{2} \times 0.6745}\right] \approx \exp (0.95 \tau)
$$

where $\tau^{2}$ is the hospital-level variance. If the MOR is 1 , there is no variation between hospitals. If there is considerable between-hospital variation, the MOR will be large.

Because the two measures of inter-hospital variation, $\tau^{2}$ and ICC, are difficult to interpret [31] the MOR is considered as an alternative measure. While ICC and MOR have a direct relationship due to their shared basis on $\tau^{2}$, the different functions of that inter-hospital variation give rise to usefully distinct interpretations.

\section{The $80 \%$ interval odds ratio (IOR-80\%)}

The interpretation of hospital-level effects in multilevel model has been highlighted as problematic. In multilevel models, contrary to patient-level risk factors, hospitallevel risk factors only take one value in each hospital and, consequently, it is necessary to compare patients from different hospitals to quantify hospital-level associations with outcome $[41,42]$. A multilevel model odds ratio for a hospital-level risk factor needs to be interpreted as the effect of the risk factor given a comparison between two hospitals of identical random effect value whose mortality probabilities differ only with regard to the risk factor under consideration. 
To interpret effects of hospital-level risk factors more generally, the unexplained between-hospital variability needs to be taken into account. The IOR-80\% achieves this by incorporating both the fixed hospital-level risk factor effect and the unexplained between-hospital heterogeneity in an interval. IOR-80\% shows the impact of hospital-level variables on mortality when comparing hospitals with different $u_{i}$ values.

To understand the calculation of the IOR- $80 \%$, consider all possible pairs of patients with identical patient-level risk factors from different hospitals but who differ by one unit in a hospital-level risk factor (e.g. one patient in a teaching hospital, the other in a non-teaching hospital). For each pair, the OR between the two patients is calculated, thereby obtaining a distribution of ORs. The IOR$80 \%$ is defined as the interval around the median of the distribution that comprises $80 \%$ of the OR values. In practice, the lower and upper bounds of the IOR- $80 \%$ can be computed using the approximation

$$
\operatorname{IOR}-80 \% \frac{\text { lower }}{\text { upper }}=\exp \left[\beta \pm 1.2816 \sqrt{2 \times\left(\tau^{2}\right)}\right] \approx \exp (\beta \pm 1.81 \tau)
$$

where $\beta$ is the regression coefficient for the hospitallevel variable, $\tau^{2}$ is the hospital-level variance, and the values -1.2816 and +1.2816 are respectively the 10 th and 90th centiles of the standard normal distribution.

From equation (5), a small amount of between hospital variation, $\tau^{2}$, will lead to a narrow IOR, whereas large $\tau^{2}$ leads to wider intervals. The combination of $\tau^{2}$ with the effect of the hospital-level risk factors in (5), indicates that the IOR- $80 \%$ will contain 1 if the value of $\tau^{2}$ is large compared to the effect of the hospital-level risk factors.

\section{Model estimation}

To fit the ordinary logistic regression model, maximumlikelihood was used with the Stata [43] (version 11) logistic command. The marginal logistic regression model was fitted with Stata's xtgee command and multilevel models were fitted using adaptive quadrature with 12 integration points to evaluate and maximize the marginal log likelihood by Stata's xtlogit command.

\section{Results}

The cohort consisted of 32,354 patients on the ASCTS registry admitted to 18 hospitals from 2001-2009. Patient mean age was 65.5 years (SD 12.5); $27.7 \%$ were female. Table 1 includes further patient characteristics. The number of cardiac surgeries ranged from 151 to 5314 across hospitals (Table 2). Figure 1 shows that variation in mortality rates across hospitals was considerable with rates in the range 1.0 to $4.1 \%$.

Table 3 contains the odds ratios and $95 \%$ CIs for the effects of patient and hospital characteristics from the ordinary, marginal and multilevel logistic regression
Table 1 Patient characteristics from a cardiac surgery registry 2001-2009

\begin{tabular}{|c|c|c|}
\hline Patient characteristics & Frequency & Percent \\
\hline \multicolumn{3}{|l|}{ Age (years) } \\
\hline$<60$ & 8954 & 27.6 \\
\hline 60 to 70 & 9434 & 29.2 \\
\hline 70 to 80 & 10695 & 33.1 \\
\hline $80+$ & 3271 & 10.1 \\
\hline \multicolumn{3}{|l|}{ Gender } \\
\hline Male & 23385 & 72.3 \\
\hline Female & 8969 & 27.7 \\
\hline \multicolumn{3}{|c|}{ The New York heart association class } \\
\hline$|\&| \mid$ & 22470 & 69.4 \\
\hline III & 7245 & 22.4 \\
\hline IV & 2639 & 8.2 \\
\hline \multicolumn{3}{|l|}{ Urgency of procedure } \\
\hline Elective & 19869 & 61.4 \\
\hline Urgent & 10665 & 33.0 \\
\hline Emergency/salvage & 1807 & 5.6 \\
\hline \multicolumn{3}{|l|}{ Ejection fraction estimate (\%) } \\
\hline Mild $(>45)$ & 21919 & 80.0 \\
\hline Moderate (30-45) & 3828 & 14.0 \\
\hline Severe $(<30)$ & 1656 & 6.0 \\
\hline Hypercholesterolaemia & 22072 & 68.6 \\
\hline Preoperative dialysis & 523 & 1.6 \\
\hline Previous cardiac surgery & 2776 & 8.6 \\
\hline \multicolumn{3}{|l|}{ Procedure type } \\
\hline CABG & 19907 & 61.6 \\
\hline Valve(s) & 4571 & 14.1 \\
\hline Valve(s)+CABG & 3367 & 10.4 \\
\hline Other & 4472 & 13.8 \\
\hline Inotropic medication & 888 & 2.8 \\
\hline Peripheral vascular disease & 3583 & 11.1 \\
\hline \multicolumn{3}{|l|}{ Body mass index $\left(\mathrm{kg} / \mathrm{m}^{2}\right)$} \\
\hline$<25$ & 9077 & 28.1 \\
\hline $25+$ & 23277 & 71.9 \\
\hline
\end{tabular}

models. Overall the 95\% CIs for hospital-level variables in the marginal and multilevel model were wider than in the ordinary logistic regression, reflecting the betweenhospital heterogeneity that is erroneously not accounted for in the latter model (Figure 2). In particular the effect of median annual number of cardiac surgeries was statistically significant in ordinary logistic regression, but not in the other models.

In the marginal model the correlation between mortality outcomes for any two patients from the same hospital was $\rho=0.002$ suggesting a weak positive association. The multilevel model estimated that the proportion of the variance in 30 -day mortality between hospitals was $1 \%$ $(\mathrm{ICC}=0.01)$. From the multilevel model it was estimated that if a patient moved to another hospital with a higher probability of mortality, the median increase in their 
Table 2 Total number of cardiac surgeries, median of the annual number of cardiac surgeries, number of deaths within 30-days of surgery, and mortality rates in 18 hospitals 2001-2009 by academic affiliation status

\begin{tabular}{|c|c|c|c|c|}
\hline Hospital & The median annual number of cardiac surgeries & No. of surgeries* & No. of deaths & Mortality rate (\%) \\
\hline \multicolumn{5}{|l|}{ Teaching } \\
\hline 1 & 310 & 1174 & 37 & 3.15 \\
\hline 2 & 163 & 326 & 10 & 3.07 \\
\hline 3 & 195 & 780 & 8 & 1.03 \\
\hline 4 & 147 & 294 & 6 & 2.04 \\
\hline 5 & 147 & 470 & 17 & 3.62 \\
\hline 6 & 293 & 1083 & 44 & 4.06 \\
\hline 7 & 209 & 418 & 8 & 1.91 \\
\hline 8 & 512 & 4361 & 176 & 4.04 \\
\hline 9 & 437 & 3729 & 123 & 3.30 \\
\hline 10 & 418 & 3291 & 129 & 3.92 \\
\hline 11 & 284 & 1131 & 27 & 2.39 \\
\hline 12 & 332 & 2753 & 93 & 3.38 \\
\hline 13 & 402 & 3206 & 106 & 3.31 \\
\hline 14 & 649 & 5314 & 97 & 1.83 \\
\hline 15 & 324 & 1291 & 28 & 2.17 \\
\hline \multicolumn{5}{|c|}{ Non-teaching } \\
\hline 16 & 475 & 1723 & 47 & 2.73 \\
\hline 17 & 75 & 151 & 4 & 2.65 \\
\hline 18 & 172 & 859 & 23 & 2.68 \\
\hline
\end{tabular}

* Not all hospitals reported for all 9 years in the period 2001-2009

odds of mortality would be 1.2 -fold $(\mathrm{MOR}=1.2)$, a modest effect compared to patient-level risk factor effects in Table 2 but comparable to the hospital-level fixed effects in Figure 2.

Interpretation of the hospital-level effects estimated from marginal logistic regression $(\mathrm{OR}=1.2,95 \% \mathrm{CI}$, (0.9-1.5))was that, on average, the odds of mortality for patients in teaching hospitals increased by $20 \%$ compared to that of patients in non-teaching hospitals. In

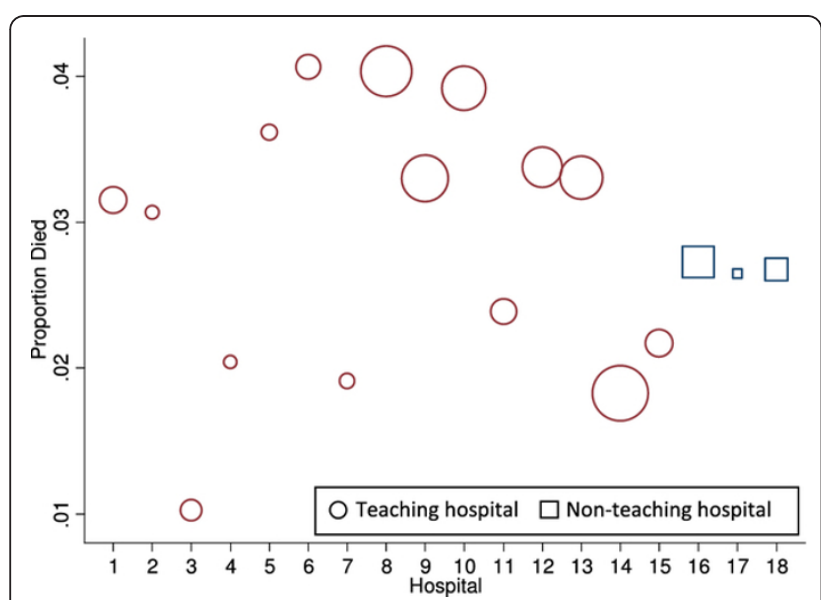

Figure 1 Mortality within 30-days of cardiac surgery in teaching and non-teaching hospitals. The area of their circle is proportional to the number of surgeries in each of the 18 hospitals. comparison, the multilevel logistic regression odds ratio of $1.3,95 \% \mathrm{CI},(0.8-1.9)$ for the same parameter says that if comparing two patients with identical risk factors, one treated in a teaching hospital and one treated in a nonteaching hospital, and with those hospitals otherwise identical with regard to mortality risk, then the odds of mortality was increased 1.3-fold for the patient in the teaching hospital. The magnitude of these effects may be of high importance clinically but the difficulty in interpretation, particularly with regard to the existence of hospitals with identical underlying mortality risk, may limit their usefulness.

The IOR-80\% for academic affiliation was 0.8 to 1.8 which provides the further insight that, when comparing two randomly chosen patients with identical risk factors, one from a teaching hospital, the other from a nonteaching hospital, and those hospitals possibly differing in other ways regarding mortality risk, the odds ratio for the comparison will, with $80 \%$ probability, lie between 0.8 and 1.8. In other words, even disregarding the uncertainty inherent in sampling that can be incorporated in confidence intervals, the wide IOR-80\% reflects considerable uncertainty in the impact of hospital academic affiliation on patient-level mortality risk due to substantial residual variation in mortality between hospitals that was not accounted for by either academic affiliation or median annual number of cardiac surgeries or patientlevel characteristics included in the regression model. 
Table 3 Ordinary, marginal and multilevel logistic regression results: Odds ratios, OR, describing associations with 30-day mortality for patient-level and hospital-level characteristics

\begin{tabular}{|c|c|c|c|c|c|c|}
\hline \multirow[t]{2}{*}{ Risk factor } & \multicolumn{2}{|c|}{ Ordinary } & \multicolumn{2}{|c|}{ Marginal } & \multicolumn{2}{|c|}{ Multilevel* } \\
\hline & OR & $95 \% \mathrm{Cl}$ & OR & $95 \% \mathrm{Cl}$ & OR & $95 \% \mathrm{Cl}$ \\
\hline \multicolumn{7}{|l|}{ Patient-level } \\
\hline \multicolumn{7}{|l|}{ Age group ( $<60$ y, reference group): } \\
\hline $60-70$ y & 1.5 & $(1.2,2.0)$ & 1.5 & $(1.2-1.8)$ & 1.5 & $(1.2,2.0)$ \\
\hline $70-80$ y & 2.7 & $(2.1,3.4)$ & 2.4 & $(2.0-3.0)$ & 2.7 & $(2.1,3.3)$ \\
\hline $80+y$ & 3.6 & $(2.8,4.8)$ & 3.3 & $(2.7-4.5)$ & 3.7 & $(2.8,4.9)$ \\
\hline Gender (female vs. male) & 1.3 & $(1.1,1.6)$ & 1.3 & $(1.1-1.5)$ & 1.3 & $(1.1,1.6)$ \\
\hline \multicolumn{7}{|l|}{ NYHA Class (I\&ll, reference group): } \\
\hline III & 1.3 & $(1.1,1.6)$ & 1.3 & $(1.1-1.6)$ & 1.4 & $(1.1,1.6)$ \\
\hline IV & 1.7 & $(1.4,2.1)$ & 1.6 & $(1.3-2.0)$ & 1.7 & $(1.4,2.2)$ \\
\hline \multicolumn{7}{|l|}{ Urgency of procedure (elective, reference group): } \\
\hline Urgent & 2.0 & $(1.6,2.4)$ & 1.8 & $(1.5-2.2)$ & 2.0 & $(1.6,2.4)$ \\
\hline Emergency/salvage & 5.4 & $(4.3,7.0)$ & 4.9 & $(4.0-6.0)$ & 5.5 & $(4.3,7.0)$ \\
\hline \multicolumn{7}{|l|}{ Ejection fraction estimate (Mild, $>45 \%$, reference group): } \\
\hline Moderate $(30-45 \%)$ & 1.4 & $(1.2,1.8)$ & 1.3 & $(3.0-1.6)$ & 1.4 & $(1.2,1.8)$ \\
\hline Severe $(<30 \%)$ & 2.3 & $(1.8,2.9)$ & 2.2 & $(1.8-2.7)$ & 2.3 & $(1.8,2.9)$ \\
\hline Hypercholesterolaemia & 1.0 & $(0.8,1.1)$ & 1.0 & $(0.8-1.1)$ & 1.0 & $(0.8,1.2)$ \\
\hline Preoperative dialysis & 2.2 & $(1.5,3.2)$ & 2.2 & $(1.5-3.0)$ & 2.2 & $(1.5,3.2)$ \\
\hline Previous cardiac surgery & 1.6 & $(1.3,2.0)$ & 1.6 & $(1.3-2.0)$ & 1.7 & $(1.3,2.0)$ \\
\hline \multicolumn{7}{|l|}{ Procedure type (CABG, reference group): } \\
\hline Valve(s) & 1.7 & $(1.3,2.2)$ & 1.6 & $(1.2-2.0)$ & 1.7 & $(1.3,2.2)$ \\
\hline Valve(s)+CABG & 2.2 & $(1.8,2.8)$ & 2.0 & $(1.6-2.6)$ & 2.2 & $(1.8,2.8)$ \\
\hline Other & 3.4 & $(2.7,4.1)$ & 3.0 & $(2.4-3.7)$ & 3.4 & $(2.8,4.2)$ \\
\hline Inotropic medication & 3.1 & $(2.4,3.9)$ & 3.0 & $(2.4-3.7)$ & 3.2 & $(2.5,4.1)$ \\
\hline Peripheral vascular disease & 1.7 & $(1.4,2.1)$ & 1.6 & $(1.3-2.0)$ & 1.7 & $(1.4,2.0)$ \\
\hline Body mass index $\left(25+\mathrm{vs} .<25 \mathrm{~kg} / \mathrm{m}^{2}\right)$ & 0.8 & $(0.7,1.0)$ & 0.9 & $(0.7-1.0)$ & 0.8 & $(0.7,1.0)$ \\
\hline \multicolumn{7}{|l|}{ Hospital-level } \\
\hline Academic affiliation (teaching vs. non-teaching) & 1.2 & $(0.9,1.7)$ & 1.2 & $(0.9-1.5)$ & 1.3 & $(0.8,1.9)$ \\
\hline Median of the annual number of cardiac surgeries 2001-2009, per 100 surgeries & 0.88 & $(0.83,0.94)$ & 0.93 & $(0.9-1.0)$ & 0.91 & $(0.82,1.1)$ \\
\hline
\end{tabular}

*The estimated hospital-level variance, $\tau^{2}$, was 0.04 .

*The estimated hospital-level variance, $\tau^{2}$, was 0.04

The IOR- $80 \%$ for the median of the annual number of cardiac surgeries was 0.6 to 1.3 . Hence when comparing two randomly chosen patients with identical risk factors except for treatment at respective hospitals which differed by 100 in their median annual number of cardiac surgeries, and possibly differing in $u_{i}$ values, the odds ratio for the comparison will, with $80 \%$ probability, lie between 0.6 and 1.3. As for academic affiliation, this is a wide IOR- $80 \%$.

\section{Discussion}

This paper examined the application and interpretation of ordinary, marginal and multilevel logistic regression for explaining between-hospital heterogeneity in 30-day mortality outcomes following cardiac surgery in Australian hospitals.

While this paper focused on the three measures, ICC, MOR and IOR_80\%, there are other measures of variance and clustering. Alternating logistic regression [44], ALR, is a method for a statistical index of patient clustering in the form of pair-wise odds ratio. The proportional change in variance [45] is another measure that could be used for explaining variance across hospitals by patient characteristics. While we interpret $\rho$ as an estimate of ICC, this correlation between pairs of binary outcomes can be of particular interest in twin or longitudinal data [46]. The relative strengths of ALR, multilevel and marginal logistic regression have been examined by others [47].

Appropriately reflecting the between-hospital heterogeneity, the 95\% confidence intervals for hospital-level variables in the marginal and multilevel model were wider than in the ordinary logistic regression. The MOR translated the impact of the between-hospital residual variability to an effect describing relative mortality risk of patients form different hospitals. The IOR-80\% for 


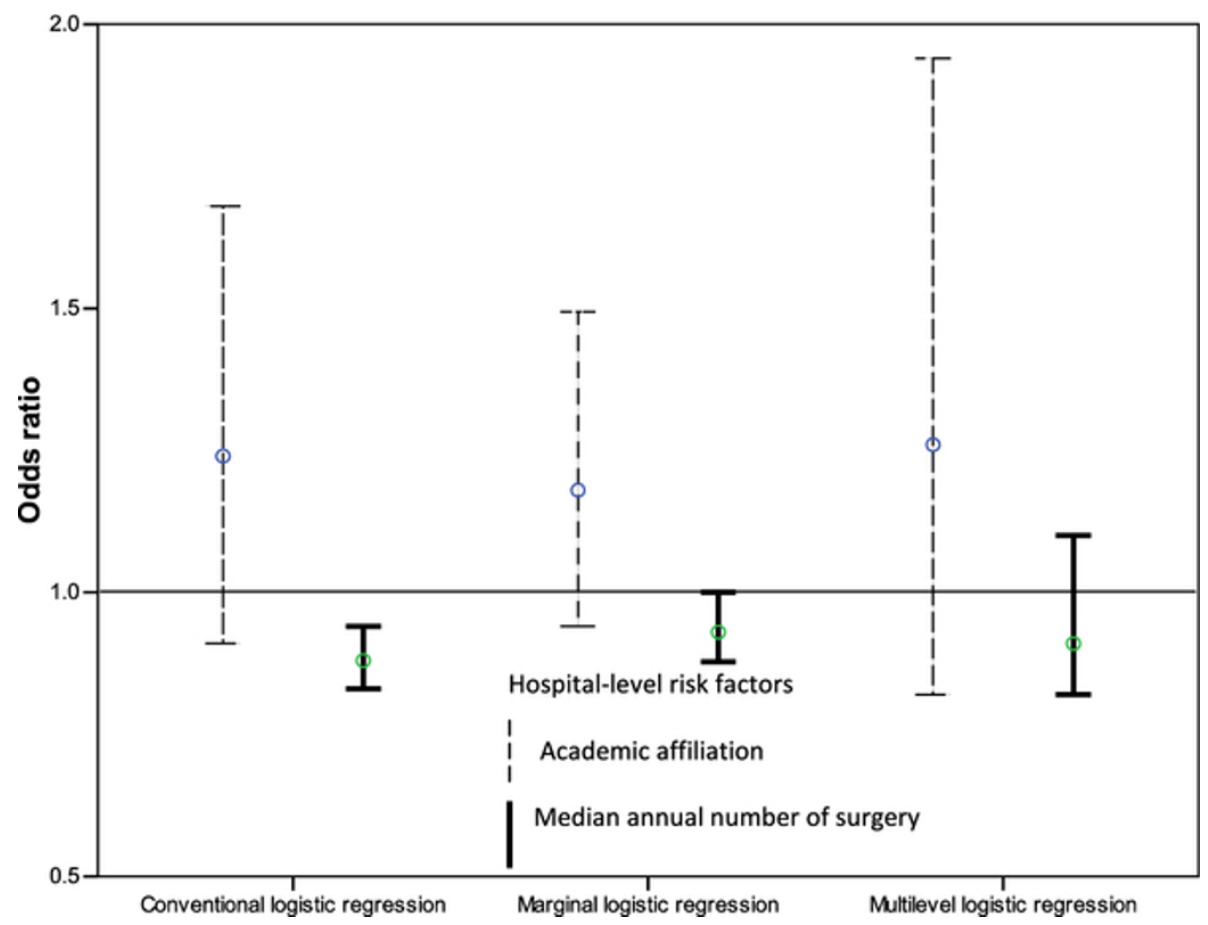

Figure 2 Odds ratio estimates and $95 \% \mathrm{Cls}$ derived from conventional, marginal and multilevel logistic regression for 30-day mortality for academic affiliation and median annual number of surgery.

both academic affiliation and median annual of cardiac surgeries were relatively wide, reflecting the large unexplained variation between hospitals in mortality. The inclusion of OR = 1 in both intervals suggested an inability of hospital-level risk factors to add meaningfully to explaining variation in mortality rates.

Marginal models do not require any distributional assumptions beyond correctly modelling the mean (average) outcome, yet when combined with robust standard errors they can provide appropriate inferences. They estimate within-hospital dependency, but do not directly estimate variance components. A methodological limitation of relevance here is that the robust standard errors are underestimated by marginal models for studies with a small number of hospitals, especially if the numbers of patients per hospital are severely unbalanced. Using bootstrap methods for estimating standard errors is an alternative approach [41,48-50].

In contrast, the most straightforward interpretation of hospital to hospital variability in mortality came from the MOR in the multilevel model. Being on the odds ratio scale, the MOR allows unexplained hospital to hospital variability to be directly compared with patientlevel and hospital-level risk factor effects. Using IOR$80 \%$ for measuring the association between the hospitallevel risk factors and mortality, the between hospital heterogeneity becomes relevant for understanding the real impact of hospital-level risk factors.
In previous studies, for assessing hospital-level characteristics on mortality, ordinary and multilevel logistic regression were compared but ICC, MOR and IOR-80\% were not reported,[51,52] or only ICC provided [23].

Multilevel and marginal methods address different questions and the choice of method needs to be made according to the research objective. Multilevel models are best equipped to address questions relating to modification of a particular hospital [29]. A marginal approach does not make specific use of within-hospital information for hospital-level covariates [29]. For example, in a study assessing hospital-level characteristics on mortality after acute myocardial infarction the OR point estimates were of interest more than their statistical significance, so an ordinary logistic regression model was applied for its greater familiarity and hence comprehensibility [53]. The marginal model is recommended if the aim is estimation of the effects of patient-level risk factors while adjusting for between hospital heterogeneity. Marginal logistic regression with the exchangeable working correlation matrix has been used for patients within surgical centres [26] where the study goal was to determine the relationship between in-hospital mortality after coronary artery bypass graft surgery.

For specific hospital interpretation or hospital ranking the multilevel model is recommended [54,55]. In a study for comparing neonatal mortality in low- and high-risk deliveries in different hospitals MOR and IOR-80\% were 
reported [56] however use of these two statistics has not extended to cardiac surgery outcome analysis.

\section{Conclusion}

This paper outlines the application and interpretation of marginal and multilevel modelling to patient-level cardiac surgery outcomes observed across multiple hospitals. The interpretation of hospital-level risk factors differs between methods and the subtleties of the interpretations have been clarified.

Choosing between a marginal or multilevel model depends on the goals of the analyses. Knowing the assumptions of each method and how these assumptions affect the inferences from the analysis will enable researchers to determine the best approach to analysing their data.

\section{Acknowledgements}

We thank the following staff of the ASCTS Data Management Centre,

Monash University for practical assistance: Dr Lavinia Tran, Dr Diem Dinh, Ms Angela Brennan. We acknowledge the essential role of the members of the ASCTS Database Program Steering Committee in the ongoing existence of the registry and facilitating its use in our project: Mr Gil Shardey (Chair), Mr Peter Skillington, Mr Julian Smith, Mr Andrew Newcomb, Mr Siven Seevanayagam, Mr Bo Zhang, Mr Hugh Wolfenden, Mr Adrian Pick, Prof Chris Reid, Dr Lavinia Tran, Dr Diem Dinh, Mr Andrew Clarke.

The Australasian Society of Cardiac and Thoracic Surgeons (ASCTS) National Cardiac Surgery Database Program is funded by the Department of Health Victoria, and the Health Administration Corporation (GMCT) and the Clinical Excellence Commission (CEC), NSW.

The following investigators, data managers and institutions participated in the ASCTS Database: Alfred Hospital: Pick A, Duncan J; Austin Hospital: Seevanayagam S, Shaw M; Cabrini Health: Shardey G; Geelong Hospital: Morteza M, Zhang B, Bright C; Flinders Medical Centre: Knight J, Baker R, Helm J, Canning N; Jessie McPherson Private Hospital: Smith J, Baxter H; Hospital: John Hunter Hospital: James A, Scaybrook S; Lake Macquarie Hospital: Dennett B, Mills M; Liverpool Hospital: French B, Hewitt N; Mater Health Services: Diqer AM, Curtis L; Monash Medical Centre: Smith J, Baxter $\mathrm{H}$; Prince of Wales Hospital: Wolfenden H, Weerasinge D; Royal Melbourne Hospital: Skillington P, Law S; Royal Prince Alfred Hospital: Wilson M, Turner L; St George Hospital: Fermanis G, Newbon P; St Vincent's Hospital, VIC: Yii M, Newcomb A, Mack J, Duve K; St Vincent's Hospital, NSW: Spratt P, Hunter T; The Canberra Hospital: Bissaker P, Bhosale M; Townsville Hospital: Tam R, Farley A; Westmead Hospital: Costa R, Halaka M.

\section{Authors' contributions}

MS designed and conducted the study, performed the statistical analysis, wrote the draft of the manuscript to which all authors subsequently contributed. RW and AF contributed to the design and conduct of the study CMR was responsible for the data collection process and issues related to data quality. All authors read and revised the manuscript for important intellectual content and approved the final manuscript.

\section{Competing interests}

The authors declare that they have no competing interests.

Received: 13 April 2011 Accepted: 12 March 2012

Published: 12 March 2012

\section{References}

1. Alter DA, Naylor CD, Austin PC, Tu JV: Long-term MI outcomes at hospitals with or without on-site revascularization. JAMA 2001, 285:2101-2108.

2. Thiemann DR, Coresh J, Oetgen WJ, Powe NR: The association between hospital volume and survival after acute myocardial infarction in elderly patients. N Engl J Med 1999, 340:1640-1648.
3. Jollis JG, DeLong ER, Peterson ED, Muhlbaier LH, Fortin DF, Califf RM, Mark DB: Outcome of acute myocardial infarction according to the specialty of the admitting physician. N Engl J Med 1996, 335:1880-1887.

4. Every NR, Larson EB, Litwin PE, Maynard C, Finn SD, Eisenberg MS, Hallstrom AP, Martin JS, Weaver WD: The association between on-site cardiac catheterization facilities and the use of coronary angiography after acute myocardial infarction. N Engl J Med 1993, 329:546-551.

5. Krumholz HM, Chen J, Murillo JE, Cohen DJ, Radford MJ: Admission to hospitals with on-site cardiac catheterization facilities. Circulation 1998, 98:2010-2016.

6. Wilson DJ, Soumerai SB, Palmer RH: Association of physician and hospital volume with use of aspirin and reperfusion therapy in acute myocardial infarction. Medical Care 2000, 38:1092-1102.

7. Hannan EL, Racz M, Ryan TJ, McCallister BD, Johnson LW, Arani DT, Guerci AD, Sosa J, Topol EJ: Coronary angioplasty volume-outcome relationships for hospitals and cardiologists. JAMA 1997, 277:892-898.

8. Jollis JG, Peterson ED, Nelson CL, Stafford JA, DeLong ER, Muhlbaier LH, Mark DB: Relationship between physician and hospital coronary angioplasty volume and outcomes in elderly patients. Circulation 1997, 95:2485-2491.

9. Rothman KJ, Greenland S: Modern epidemiology. 2 edition. Philadelphia: Lippincott Williams \& Wilkins; 1998.

10. Hosmer DW, Lemeshow S: Applied logistic regression New York: John Wiley \& Sons; 1989.

11. McCullagh P, Nelder JA: Generalized linear models. 2 edition. London: Chapman \& Hall; 1989.

12. Duncan C, Jones K, Moon G: Context, composition and heterogeneity: using multilevel models in health research. Soc Sci Med 1998, 46:97-117.

13. Rice N, Leyland A: Multilevel models: applications to health data. J Health Serv Res Policy 1996, 1(3):154-164.

14. Demidenko E: Mixed models: theory and applications London: WileyInterscience; 2004.

15. Goldstein H: Multilevel statistical models. 2 edition. New York: Edward Arnold; 1995.

16. Snijders TAB, Bosker RJ: Multilevel analysis: an introduction to basic and advanced multilevel modeling London: Sage Publications; 1999.

17. McCulloch CE, Searle SR: Generalized, Linear, and Mixed Models. 2 edition. New York: John Wiley; 2001.

18. Diggle PJ, Liang KY, Zeger SL: Analysis of longitudinal data: Oxford England: Clarendon Press; 1994.

19. Fitzmaurice GM, Laird NM, Ware JH: Applied longitudinal analysis Philadelphia: Wiley-Interscience; 2004.

20. Donner A, Klar N: Design and analysis of cluster randomization trials in health research London, United Kingdom: Arnold; 2000.

21. Myers RH: Classical and modern regression with applications. 2 edition. Belmont (Calif): Duxbury Press; 1990.

22. Kleinbaum DG: Logistic regression: a self-learning text New York: Springer; 1994.

23. Austin PC, Tu JV, Alter DA: Comparing hierarchical modeling with traditional logistic regression analysis among patients hospitalized with acute myocardial infarction: Should we be analyzing cardiovascular outcomes data differently? Am Heart J 2003, 145(1):27-35.

24. Austin PC, Goel V, Walraven CV: An introduction to multilevel regression models. Can J Public Health 2001, 92(2):150-154.

25. Glance LG, Dick A, Osler TM, Li Y, Mukamel DB: Impact of changing the statistical methodology on hospital and surgeon ranking the case of the New York state cardiac surgery report card. Medical Care 2006, 44:311-319.

26. Guru V, Tu JV, Etchells E, Anderson GM, Naylor CD, Novick RJ, Feindel CM, Rubens FD, Teoh K, Mathur A, et al: Relationship between preventability of death after coronary artery bypass graft surgery and all-cause riskadjusted mortality rates. Circulation 2008, 117:2969-2976.

27. Heede KVD, Lesaffre E, Diya L, Vleugels A, Clarke SP, Aiken LH, Sermeus W: The relationship between inpatient cardiac surgery mortality and nurse numbers and educational level: analysis of administrative data. Int I Nurs Stud 2009, 46:796-803.

28. Cho SH: Using multilevel analysis in patient and organizational outcomes research. Nurs Res 2003, 52(1):61-65.

29. Neuhaus JM, Kalbfleisch JD, Hauck WW: A comparison of cluster-specific and population-averaged approaches for analyzing correlated binary data. Int Stat Rev 1991, 59(1):25-36. 
30. Hu FB, Goldberg J, Hedeker D, Flay BR, Pentz MA: Comparison of population-averaged and subject-specific approaches for analyzing repeated binary outcomes. Am J Epidemiol 1998, 147(7):694-703.

31. Larsen K, Merlo J: Appropriate assessment of neighborhood effects on individual health: integrating random and fixed effects in multilevel logistic regression. Am J Epidemiol 2005, 161(1):81-88.

32. Larsen K, Petersen JH, Budtz-Jørgensen E, Endahl L: Interpreting parameters in the logistic regression model with random effects. Biometrics 2000, 56(3):909-914.

33. Reid CM, Rockell M, Skillington PD, Shardey GC, Smith JA, Yii M, Seevanayagam S, Mohajeri M, Rowland M: Initial twelve months experience and analysis for 2001-2002 from the australasian society of cardiac and thoracic surgeons-victorian database project. Heart Lung Circ 2004, 13(3):291-297.

34. Billah B, Reid CM, Shardey GC, Smith JA: A preoperative risk prediction model for 30-day mortality following cardiac surgery in an Australian cohort. Eur J Cardiothorac Surg 2010, 37:1086-1092.

35. Cox DR, Snell JE: Analysis of binary data. 2 edition. London: CRC/Chapman \& Hall; 1989

36. Liang KY, Zeger SL: Longitudinal data analysis using generalized linear models. Biometrika 1986, 73(1):13-22.

37. Hubbard AE, Ahern J, Fleischer NL, Laan MVd, Lippman SA, Jewell N, Bruckner T, Satariano WA: To GEE or not to GEE: comparing population average and mixed models for estimating the associations between neighborhood risk factors and health. Epidemiology 2010, 21:467-474.

38. Kim HY, Preisser JS, Rozier RG, Valiyaparambil JV: Multilevel analysis of group randomized trials with binary outcomes. Community Dent Oral Epidemiol 2006, 34(4):241-251.

39. Goldstein H, Browne W, Rasbash J: Partitioning variation in generalised linear multilevel models. Understand Stat 2002, 1:223-232.

40. Rasbash J, Steele F, Browne W, Prosser B: Logistic models for binary and binomial responses. A user's guide to MLwiN Version 2.0. Documentation Version 2.1e London: Centre for Multilevel Modelling, Institute of Education, University of London; 2003.

41. Merlo J, Chaix B, Ohlsson H, Beckman A, Johnell K, Hjerpe P, Rastam L, Larsen $\mathrm{K}$ : A brief conceptual tutorial of multilevel analysis in social epidemiology: using measures of clustering in multilevel logistic regression to investigate contextual phenomena. J Epidemiol Community Health 2006, 60(4):290-297.

42. Zeger SL, Liang KY, Albert PS: Models for longitudinal data: a generalized estimating equation approach. Biometrics 1988, 44(4):1049-1060.

43. StataCorp: Stata Statistical Software: Release 11. College Station, TX: StataCorp LP 2009.

44. Carey VJ, Zeger SL, Diggle P: Modelling multivariate binary data with alternating logistic regressions. Biometrika 1993, 80(3):517-526.

45. Merlo J, Yang M, Chaix B, Lynch J, Ra ${ }^{\circ}$ stam L: A brief conceptual tutorial on multilevel analysis in social epidemiology: investigating contextual phenomena in different groups of people. J Epidemiol Community Health 2005, 59(9):729-736.

46. Lipsitz SR, Fitzmaurice GM: Estimating equations for measures of association between repeated binary responses. Biometrics 1996, 52(3):903-912.

47. Preisser JS, Arcury TA, Quandt SA: Detecting patterns of occupational illness clustering with alternating logistic regressions applied to longitudinal data. Am J Epidemiol 2003, 158(5):495-501.

48. Donner A: Some aspects of the design and analysis of cluster randomization trials. J R Stat Soc 1998, 47(1):95-113.

49. Murray DM, Hannan PJ, Wofinger RD, Baker WL, Dwyer JH: Analysis of data from group-randomised trials with repeat observations on the same group. Stat Med 1998, 17:1581-1600.

50. Lindsey jK, Lambert P: On the appropriateness of marginal models for repeated measurements in clinical trials. Stat Med 1998, 17:447-469.

51. Stukenborg GJ, Wagner DP, Harrell FEJ, Oliver MN, Heim SW, Price AL, Han CK, Wolf AMD, Connors AF: Which hospitals have significantly better or worse than expected mortality rates for acute myocardial infarction patients? improved risk adjustment with present-at-admission diagnoses. Circulation 2007, 116:2960-2968.

52. Hannan EL, Wu C, DeLong ER, Raudenbush SW: Predicting risk-adjusted mortality for CABG surgery logistic versus hierarchical logistic models. Medical Care 2005, 43:726-735.
53. Rosato S, Seccareccia F, D'Errigo P, Fusco D, Maraschini A, Badoni G, Perucci CA: Thirty-day mortality after AMI: effect modification by gender in outcome studies. European Journal of Public Health 2009, 20(4):1-6.

54. D'Errigo P, Tosti ME, Fusco D, Perucci CA, Seccarecci F: Use of hierarchical models to evaluate performance of cardiac surgery centres in the Italian CABG outcome study. BMC Med Res Methodol 2007, 7:29-37.

55. Rasmussen S, Zwisler AD, Abildstrom SZ, Madsen JK, Madsen M: Hospital variation in mortality after first acute myocardial infarction in Denmark from 1995 to 2002: lower short-term and 1-Year mortality in highvolume and specialized hospitals. Medical Care 2005, 43(10):970-978.

56. Merlo J, Gerdtham UG, Eckerlund I, Hakansson S, Otterblad-Olausson P, Pakkanen M, Lindqvist PG: Hospital level of care and neonatal mortality in lowand high-risk deliveries reassessing the question in Sweden by multilevel analysis. Medical Care 2005, 43:1092-1100.

\section{Pre-publication history}

The pre-publication history for this paper can be accessed here: http://www.biomedcentral.com/1471-2288/12/28/prepub

doi:10.1186/1471-2288-12-28

Cite this article as: Sanagou et al: Hospital-level associations with 30day patient mortality after cardiac surgery: a tutorial on the application and interpretation of marginal and multilevel logistic regression. BMC Medical Research Methodology 2012 12:28.

\section{Submit your next manuscript to BioMed Central and take full advantage of:}

- Convenient online submission

- Thorough peer review

- No space constraints or color figure charges

- Immediate publication on acceptance

- Inclusion in PubMed, CAS, Scopus and Google Scholar

- Research which is freely available for redistribution

Submit your manuscript at www.biomedcentral.com/submit
Ciomed Central 